\title{
MODELO DIDÁTICO PARA ESTUDO DE TENSÕES DO SOLO E SIMULAÇÃO DE CONTENÇÕES
}

Felipe Nunes Belufi-fbelufi@gmail.com

Universidade Federal de Mato Grosso, Faculdade de Engenharia - FAENG

Av. Fernando Corrêa da Costa, $n^{\circ} 2367$

78060-900 - Cuiabá - MT

Lameck Messias Alves Costa - lameck10@outlook.com

Universidade Federal de Mato Grosso, Faculdade de Engenharia - FAENG

Av. Fernando Corrêa da Costa, $n^{\circ} 2367$

78060-900 - Cuiabá - MT

Orlando Manica Filho - orlandinho-manical @ hotmail.com

Universidade Federal de Mato Grosso, Faculdade de Engenharia - FAENG

Av. Fernando Corrêa da Costa, $n^{\circ} 2367$

78060-900 - Cuiabá - MT

Renata Conciani - orlandinho-manical@hotmail.com

Universidade Federal de Mato Grosso, Faculdade de Arquitetura, Engenharia e TecnologiaFAET

Av. Fernando Corrêa da Costa, $n^{\circ} 2367$

78060-900 - Cuiabá - MT

Resumo: A grande maioria das obras e intervenções humanas no ambiente natural se relacionam com o solo ou com o maciço rochoso, seja demandando esforços que o substrato deve resistir, ou utilizando dele como estrutura em construções, ou ainda, em ambas as situações. $O$ estudo das relações desses esforços nos solos são fundamentais desde a concepção de projetos de engenharia, até posteriormente, no monitoramento do comportamento das intervenções no meio ambiente. Devido à isto, é previsto nos currículos de engenharia o ensino da Geotecnia. Tendo em vista essas premissas, este artigo vem apresentar alguns modelos didáticos para representar os esforços relacionados ao solo, e algumas aplicação em soluções de engenharia geotécnica. Os modelos podem facilitar o entendimento das relações entre as tensões verticais e horizontais do solo, noções da organização da estrutura dos solos, além de simular algumas situações de contenção de solo, que são temas previstos nos planos de ensino de engenharias. A conclusão deste trabalho está na utilização dos modelos aos alunos de graduação, o que tem se mostrado uma ferramenta relevante para contribuir com o aprendizado dos alunos, devido à forma lúdica como é apresentada.

Palavras-chave: Geotecnia. Metodologia de ensino. Modelo reduzido.

\section{REVISÃO BIBLIOGRÁFICA}

Nesta seção, serão apresentadas as teorias referentes aos modelos didáticos. Primeiramente serão revistos os conceitos básicos sobre tensões no solo em seguida definidas as contenções de terra. 


\subsection{Tensões no solo}

Resultado do intemperismo, da desintegração mecânica ou da decomposição química, os solos são constituídos por: pedregulhos e areias, solos de partículas grossa; siltes, partículas intermediarias; e em condições especiais, as argilas que são partículas finas (CAPUTO, 1988).

Todos os materiais, inclusive os solos, de que são formados os corpos (ou meios) reais se deformam, em maior ou menor intensidade, sob a ação do seu peso próprio ou das cargas que lhes são impostas. O comportamento do material, quando carregado, depende, naturalmente, das tensões nele instaladas (CAPUTO, 1988).

A tensão vertical, em qualquer profundidade, é calculada simplesmente considerando o peso de solo acima daquela profundidade. Logo, se o peso específico do solo é constante em cada uma das camadas. A tensão vertical total pode ser calculada a partir da Equação 1 (MARINHO, 2018).

$$
\sigma \mathrm{v}=\gamma \cdot \mathrm{z}
$$

Onde:

бv: tensão vertical total em um determinado ponto do solo;

$\gamma$ : peso específico do solo;

z: profundidade do ponto analisado.

A tensão vertical em um perfil de solo ou em uma barragem, aumenta conforme a profundidade, devido ao peso próprio do material.

As tensões no solo são propagadas pelo contato partícula-partícula. Forças externas aplicadas a superfície contribuem para o desenvolvimento de tensões de cisalhamento. A determinação dessa resistência aos esforços cisalhantes nos solos estabelece uma das mais importantes análises do solo, pois esse é o critério de ruptura tradicionalmente utilizado para o solo.

Em um maciço terroso com superfície horizontal, a razão entre as tensões horizontal ( $\sigma$ h') e vertical ( $\left.\sigma v^{\prime}\right)$ efetivas é expressa por um coeficiente $\mathrm{k}_{0}$, que é constante em solos semelhantes, como expresso na Equação 2, o qual, não havendo deformação lateral, se chama coeficiente de empuxo no repouso e é representado por k0 ( CAPUTO, 1988).

$$
\mathrm{K}_{0}=\sigma \mathrm{h}^{\prime} / \sigma \mathrm{v}^{\prime}
$$

Ainda segundo o autor, se aumentando as tensões, as deformações crescem proporcionalmente, diz-se que o material se encontra no "estado elástico"; se, continuando a crescer as tensões, passam-se a observar deformações apreciáveis, revela-se o "estado plástico".

\subsection{Contenções de solo}

Contenções são obras civis construídas com a finalidade de prover estabilidade contra a ruptura de maciços e evitam o escorregamento causado pelo seu peso próprio ou por carregamentos externos. Exemplos típicos de estruturas de contenção são os muros de arrimo, as cortinas de estacas e as paredes diafragma. Todas elas são construídas para conter a possível ruptura do maciço, suportando as pressões laterais.

Talude é a denominação que se dá a qualquer superfície inclinada de um maciço de solo ou rocha. Ele pode ser natural, também denominado encosta, ou construído pelo homem, como, por exemplo, os aterros e cortes (GERSCOVICH, 2006). Talude natural é aquele que foi 
formado naturalmente pela natureza, pela ação geológica ou pela ação das intempéries (chuva, sol, vento). Os taludes construídos pela ação humana resultam de cortes em encostas, de escavações ou de lançamento de aterros. Os cortes devem ser executados com altura e inclinação adequadas, para garantir a estabilidade da obra. $\mathrm{O}$ projeto depende das propriedades geotécnicas dos materiais e das condições de fluxo. Os aterros são construídos em projetos de barragem de terra e em obras viárias e de implantação de estruturas civis, quando o solo de fundação tem baixa capacidade de suporte ou para nivelamento do terreno.

As barragens são as estruturas físicas que represam um curso de água. Essas obras têm como objetivo a criação de reservatórios, que é o acúmulo de água resultante do barramento. As finalidades podem ser diversas: abastecimento, controle de vazão, geração de energia, urbanismo, lazer, etc.

\section{METODOLOGIA}

Procurando uma alternativa para o processo de ensino e aprendizagem, para explicar o modelo de distribuição das tensões no solo, buscou-se uma forma de representação física, dentre alguns modelos já existentes. Estes últimos podem ser feitos com materiais comuns, como madeira, plástico, papel, e uma caixa de acrílico, que permitem observar o comportamento de solos, em especial os granulares, e como são geradas as tensões horizontais.

Esses modelos também servem de exemplo de simulações de barragens e de paredes de contenção. Também podem ser utilizados recursos de edição de vídeo para melhor compreensão dos fenômenos pertinentes aos solos.

Com isso decidiu-se que seria de grande valia reproduzir os experimentos desse tipo, e deixá-los a disposição dos alunos das engenharias para que possam entender melhor o comportamento dos solos.

\section{RESULTADOS E DISCUSSÕES}

Foram confeccionados dois modelos, sendo o primeiro nomeado de Do Solo, capaz de representar as partículas do solo em diversas configurações. E o segundo modelo, nomeado de Das Contenções, que por sua vez simulam diferentes tipos barreiras e contenções de solo.

\subsection{Do Solo}

Utilizou-se a madeira de um cabo de vassoura, que foi cortado de forma a destacar uma seção transversal da peça, que representa um solo granular, como a areia. Uma caixa de plástico transparente é utilizada, com volume de aproximadamente $1.000 \mathrm{~cm} 3$, e os cilindros de madeira que representam as seções transversais do solo são contidas na caixa, para demonstrar o modelo de arranjo do solo e fazer a relação da Equação 1, que apresenta uma dedução da $\sigma \mathrm{V}$.

O modelo está representado na Figura 1, e nele é possível ilustrar como as várias partículas de solo se arranjam. 
Figura 1: Modelo representativo das partículas de solo confinadas.

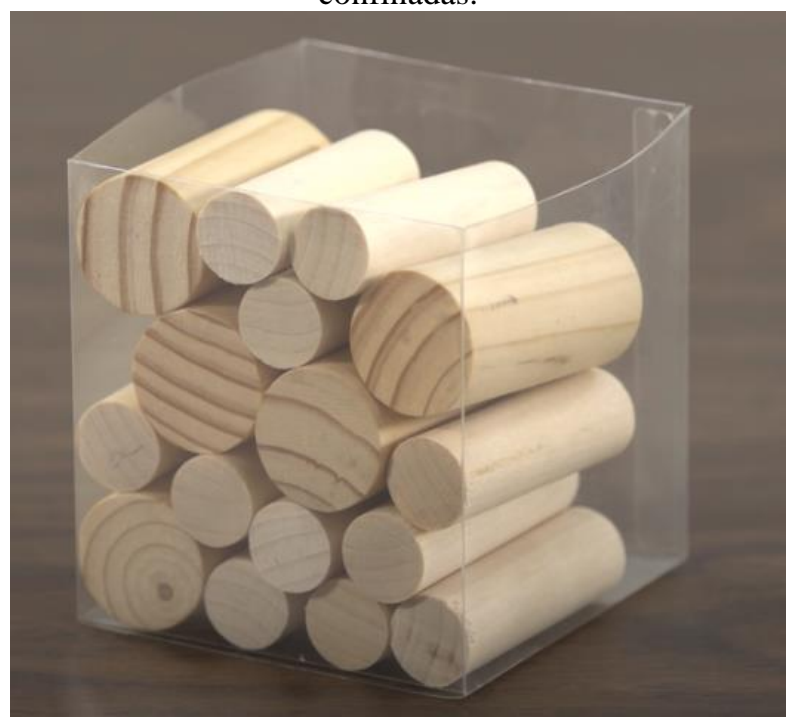

Fonte: YOUTUBE, video 6. Soil and Water Pressures.

Em seguida, os rolos cilíndricos são desconfinados da caixa plástica e apoiados lateralmente com as duas mãos, e ficam inicialmente arranjados de maneira similar de quando confinadas na caixa. Ao retirar as mãos que apoiam a lateral da composição, há a manifestação das tensões verticais/horizontais/de cisalhamento, pelo colapso da estrutura (Figura 2). Nesse questiona-se "porque isso ocorre?" e "como isso pode ocorrer no solo?".

Figura 2: Modelo de despertar das tensões em uma porção de

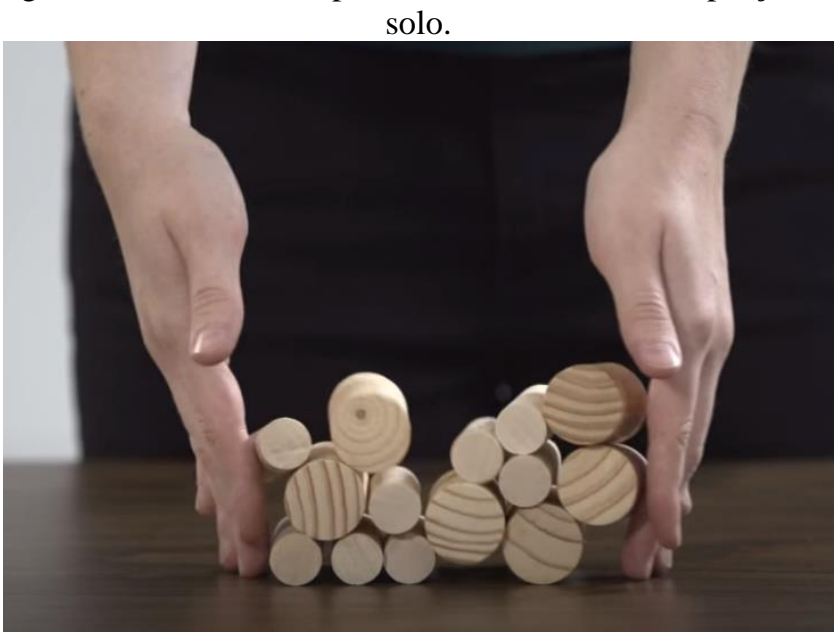

Fonte: YOUTUBE, vídeo 6. Soil and Water Pressures.

Para esclarecer essa questão, pode-se focar na relação de três partículas, como mostrado na Figura 3, representadas pelos rolos cilíndricos, demonstra-se a força gravitacional de um cilindro, atuando sob os outros dois, esses dois de deslocam na superfície horizontal. Tal demonstração enfatiza a origem das tensões horizontais, permitindo a definição do fator $\mathrm{k}$ (Equação 2), e alguns casos particulares como o k0, k=0, e k=1. 
Figura 3: Modelo da relação de três partículas, com destaque no movimento das peças.

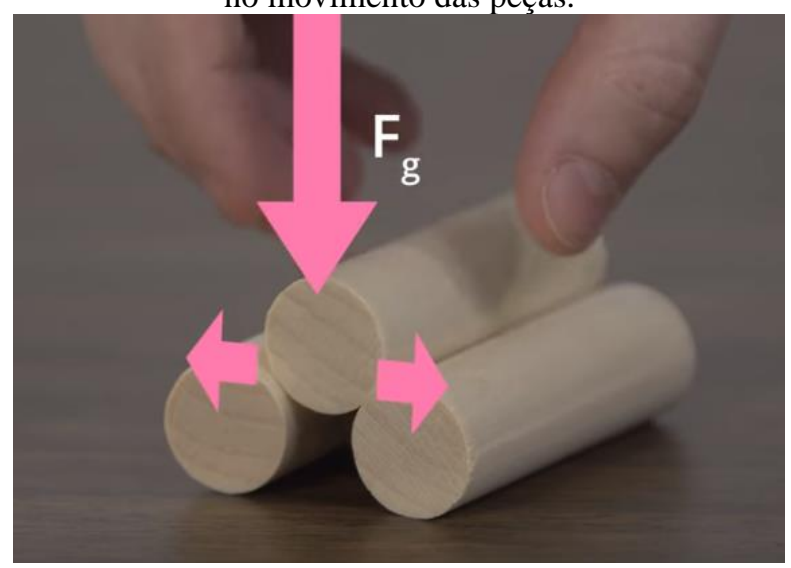

Fonte: YOUTUBE, vídeo 6. Soil and Water Pressures.

Na Figura 4 tem-se o modelo de $\mathrm{k}=0$, onde os prismas retangulares de madeira não despertam tensões horizontal, como no caso anterior e demonstram k=0.

Figura 4: Modelo de $k=0$.

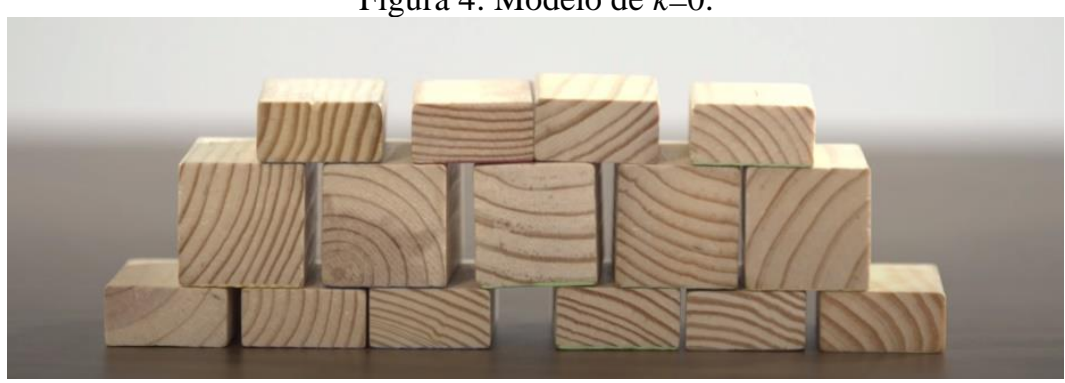

Fonte: YOUTUBE, vídeo 6. Soil and Water Pressures.

\subsection{Das Contenções}

As simulações de contenções foram possíveis com uma caixa de acrílico com dimensões $25 \mathrm{~cm}$ de comprimento e altura, e $12 \mathrm{~cm}$ de largura. A caixa de acrílico com um gabarito que apoia a membrana de papelão, mas permite que a membrana se rompa com o surgimento das tensões horizontais. O gabarito não pode interferir no movimento/momento/rompimento da parede de papelão.

Para esta simulação, são utilizadas esferas de borracha ou vidro, de $27 \mathrm{~mm}$ a $40 \mathrm{~mm}$, como a representação do solo granular em 3 dimensões, e quando são confinadas na caixa acrílica e tem a mesma capacidade de manifestar as tensões simulando o solo.

\section{Comparativo entre membrana reta e curvada}

As manifestações são medidas na comparação entre as alturas $\mathrm{z}$ até o rompimento da membrana ou das paredes.

A caixa de acrílico, as esferas e a membrana de papelão, assim como o funcionamento do experimento podem ser observados na Figura 5. 
Figura 5: Modelo de contenção com parede reta.

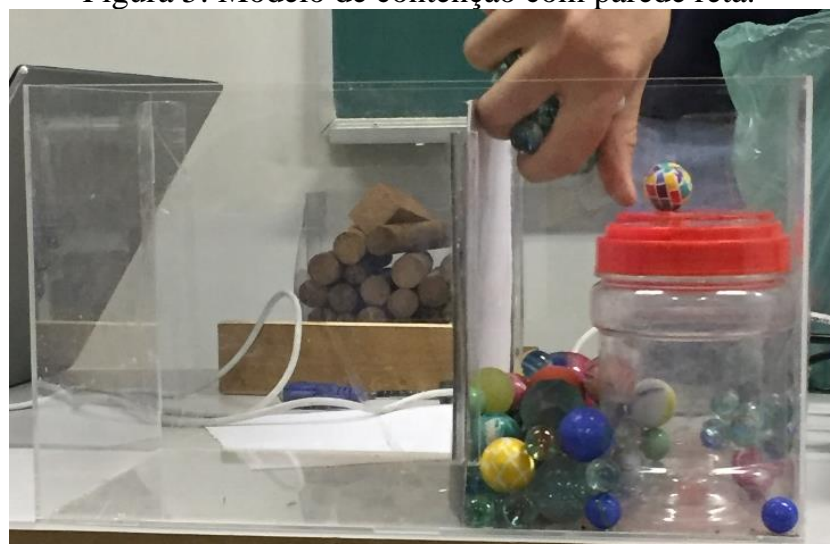

Fonte: Acervo dos autores.

Colocando a membrana de papel em posição no gabarito de forma que fique reta, à medida que mais esferas são colocadas, logo a membrana se desloca, mostrando-se incapaz de resistir aos esforços laterais. Desse modo ficando visível o primeiro local a romper sendo na parte mais funda da membrana de papel. A tensão no fundo é maior pois a maior quantidade de matéria sobreposta. Uma observação sobre o pote plástico observado na Figura 5, é que o mesmo foi utilizado apenas para fins de preenchimento de volume, devido à quantidade de esferas ter sido insuficiente para completar esse lado da caixa.

Mudando a posição da membrana de forma que forme um arco (Figura 6), fazendo a alusão com a barragem em arco, ao colocar as esferas, observa-se que ela não rompe com a mesma quantidade de esferas em relação à parede plana, suportando quase o dobro de esferas. Pois o esforço de compressão varia ao longo de seu comprimento, sendo mínimo no meio e máximos nas extremidades, aumentando a rigidez.

Figura 6: Modelo de contenção com parede arqueada com vista superior.

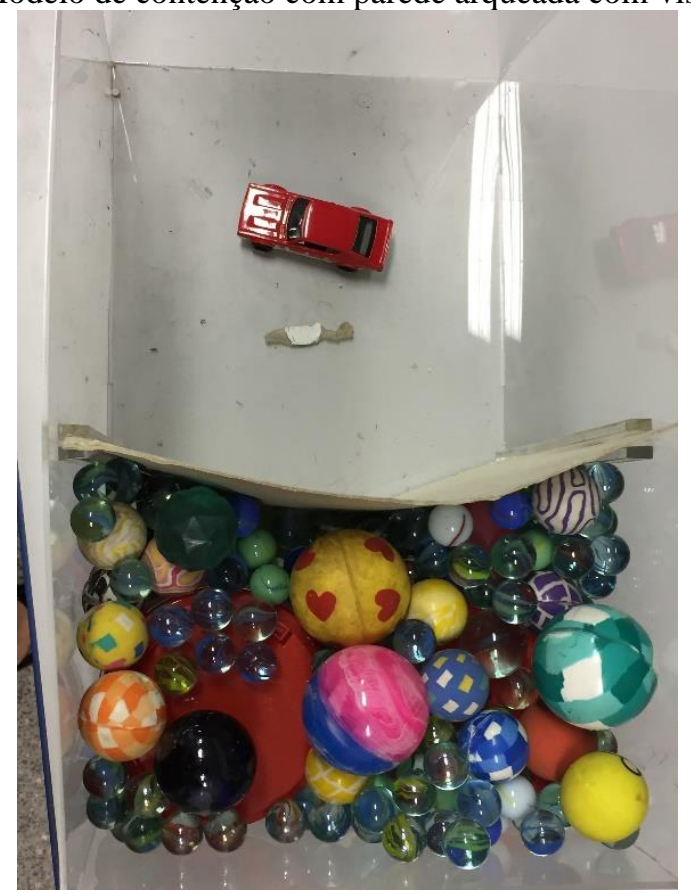

Fonte: Acervo dos autores. 
Figura 7: Comparativo visual de quantidade de esferas necessárias à ruptura da membrana de papelão.
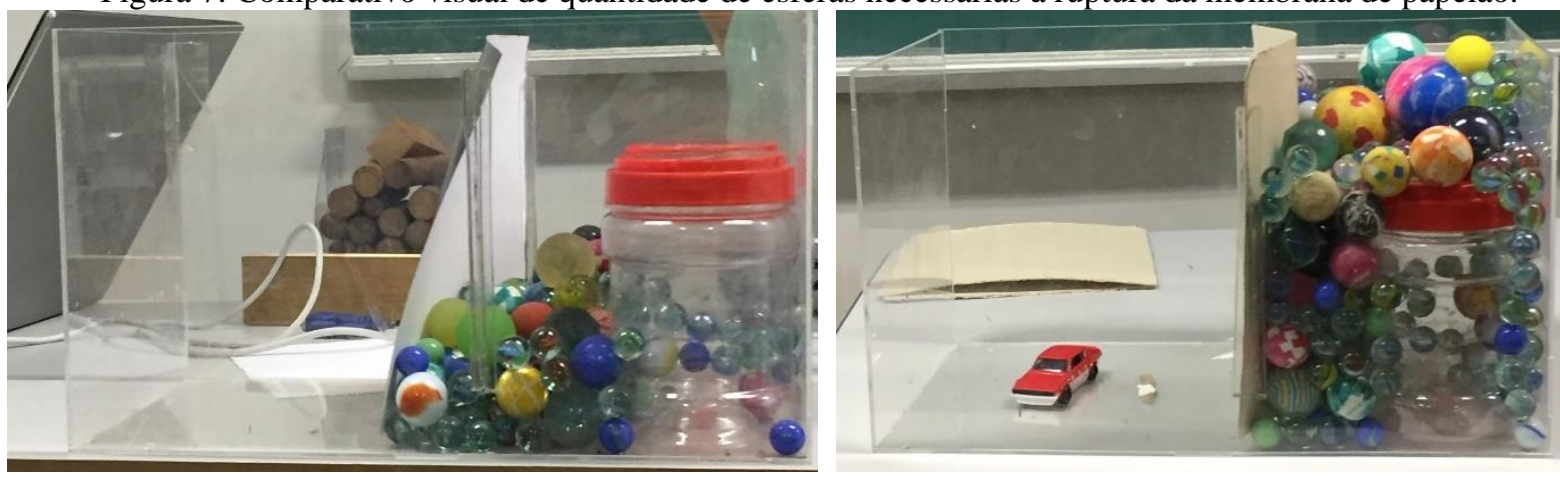

Fonte: Acervo dos autores.

\section{Simulação de Muro Cantilever}

Para simular uma parede de contenção em formato "L"' (cantilever), mostrado na Figura 8, utilizou-se um pedaço de madeira, ao invés da folha de papelão, porém com a mesma caixa de acrílico do modelo anterior.

Inicialmente apoia-se o modelo de madeira apoiada com a base para fora, e preenche-se o tardoz do "muro" com as esferas que representam o solo até se observar o colapso do sistema de contenção de madeira. Pode-se perceber a facilidade de deslocamento da peça de madeira (neste caso de rotação) que se deu devido às forças horizontais e verticais existentes devido ao peso do solo.

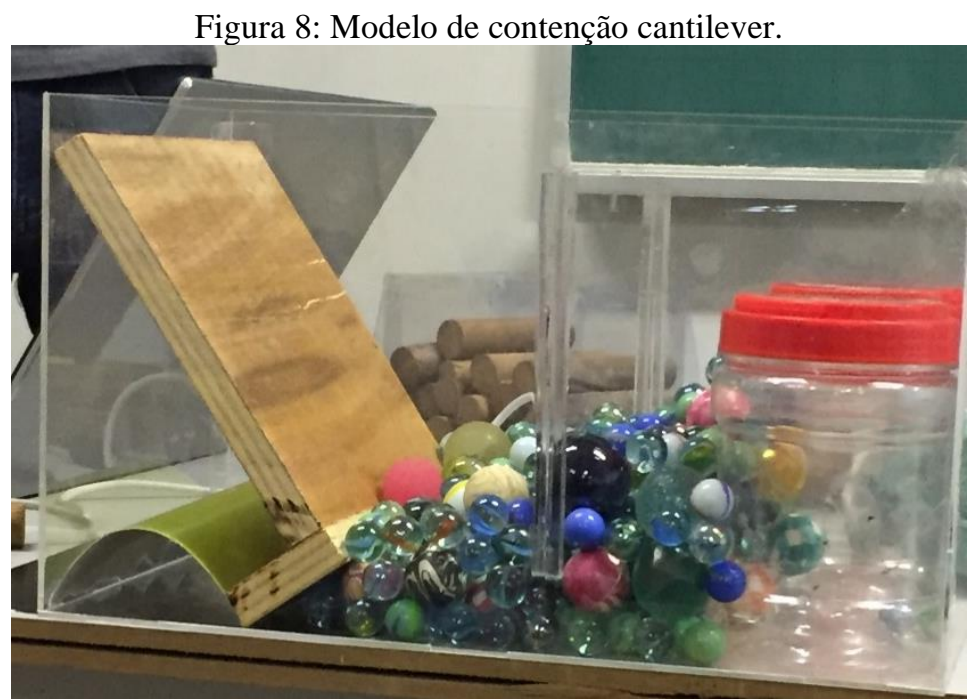

Fonte: Acervo dos autores.

Com a base da peça apontada para dentro (Figura 8), observa-se uma maior resistência ao deslocamento. A explicação se dá devido à uma nova força resistente, pois os materiais granulares geram as mesmas pressões verticais e horizontais em relação à situação anterior, mas com maior força horizontal na base, dificultando o deslocamento, que neste caso se apresentou em translação. 
Figura 9: Modelo de contenção com solo sobre a base da contenção.

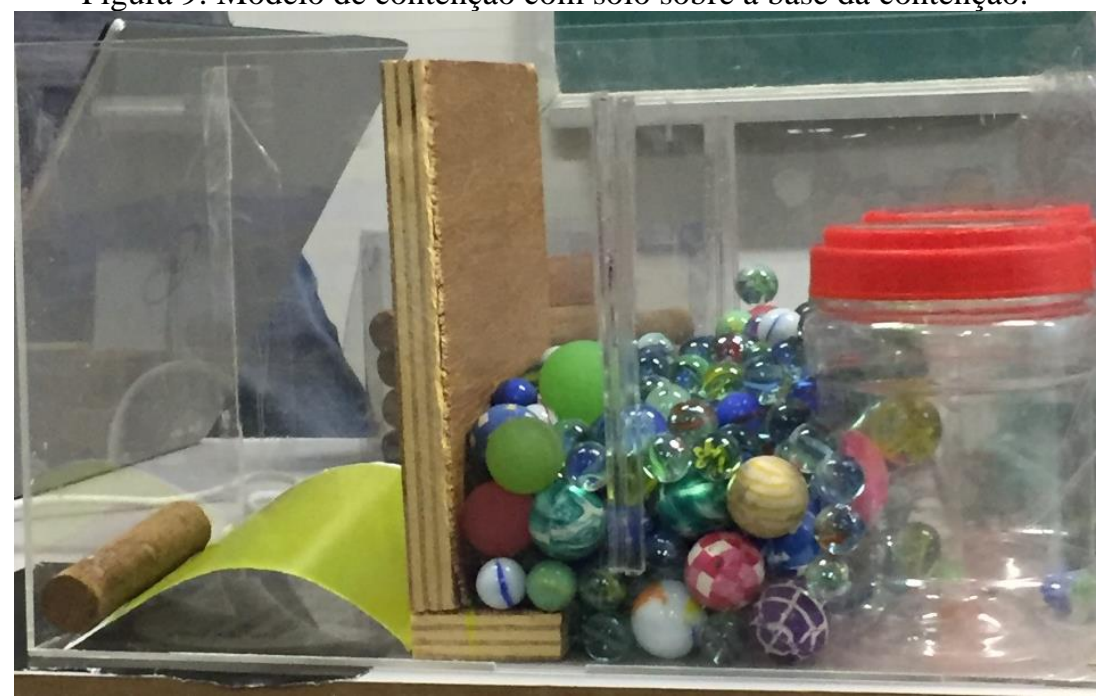

Fonte: Acervo dos autores.

\section{CONCLUSÃO E CONSIDERAÇÕES FINAIS}

As diferentes formas de analisar as tensões de solos através de experimentos simples, utilizando materiais que simulam claramente os efeitos de solo e rocha, fundamentam os efeitos de cargas aplicados sobre eles, mostrando uma comparação de como ocorre a distribuição de tensões e como elas se comportam. A utilização desse método é importante pois permite avaliar as tensões além dos limites da matemática, ou seja, sabe-se que geralmente modelos matemáticos são usados para estudos do comportamento dessas forças, e os experimentos são capazes de demonstrar através de forma visual e lúdica o comportamento dessas forças, contribuindo para capacidade de abstração de alunos de graduação no estudo desses fenômenos.

\section{REFERÊNCIAS}

BRODLAND, Prof. G. Wayne. 6. Soil and Water Pressures. Youtube, 09 mar. 2018. Disponível em < https://youtu.be/mpV8-YaJ2p4>. Acesso em: 30 jul. 19.

BRODLAND, Prof. G. Wayne. 7. Dams. Youtube, 09 mar. 2018. Disponível em < https://youtu.be/NaVCKTYQEr4>. Acesso em: 30 jul. 19.

BRODLAND, Prof. G. Wayne. 8. Retaining Walls. Youtube, 09 mar. 2018. Disponível em <https://youtu.be/iDzp6xEAT2I>. Acesso em: 30 jul. 19.

CAPUTO, Homero Pinto. Mecânica dos solos e suas aplicações: Fundamentos. 6. ed. Rio de Janeiro: Ltc, 1988. 234 p.

GERSCOVICH, Denise M. S.. Estabilidades de Taludes. 2. ed. São Paulo: Oficina de Texto, 2016.

MARINHO, Filipe. Distribuição de tensões nos solos. Disponível em: <https://www.guiadaengenharia.com/tensoes-solos-geostaticas/>. Acesso em: 20 dez. 2018. 


\title{
DIDACTIC MODEL FOR SOIL TENSIONS AND SIMULATION OF RETAINING WALLS
}

\begin{abstract}
The vast majority of human works and interventions in the environment are related to the soil, either requiring efforts that the soil must resist, or using it as a structure in constructions, or both. The study of the relationships of these efforts in soils is fundamental from the conception of engineering projects, until later, in the monitoring of the behavior of interventions in the environment. In view of these premises, this article presents some didactic models to represent the efforts related to the soil, and some applications in engineering solutions. The models can facilitate the understanding of the relationships between vertical and horizontal stresses of the soil, notions of the organization of the soil structure, in addition to simulating some situations of soil containment. The conclusion of this paper is in the presentation of the model to undergraduate students of the discipline in Soil Mechanics I and II, which proved to be a relevant tool for student learning.
\end{abstract}

Keywords: Geotechnics. Teaching Methodology. Reduced model. 